\title{
Off-shell top-pair production at NLO QCD with massive bottom quarks
}

\author{
Fabio Cascioli \\ University of Zurich \\ E-mail: cascioli@physik.uzh.ch \\ Stefan Kallweit* \\ University of Zurich \\ E-mail: kallweitephysik.uzh.ch

\section{Philipp Maierhöfer} \\ University of Zurich \\ E-mail: philipp@physik.uzh.ch
}

\section{Stefano Pozzorini}

University of Zurich

E-mail: pozzorin@physik.uzh.ch

\begin{abstract}
We report on an NLO simulation of $\mathrm{W}^{+} \mathrm{W}^{-} \mathrm{b} \overline{\mathrm{b}}$ production with massive b-quarks at the LHC. Thanks to the finite b-quark mass, $\mathrm{W}^{+} \mathrm{W}^{-} \mathrm{b} \overline{\mathrm{b}}$ predictions can be extended to the whole $\mathrm{b}$-quark phase space, thereby including Wt-channel single-top contributions that originate from collinear $\mathrm{g} \rightarrow \mathrm{b} \overline{\mathrm{b}}$ splittings, and quantum interference effects. The simulation is also applicable to exclusive 0 - and 1-jet bins, which is of great importance for Higgs-boson studies in the $\mathrm{H} \rightarrow \mathrm{W}^{+} \mathrm{W}^{-}$channel and for any other analysis with large top backgrounds and jet vetoes or jet bins.
\end{abstract}

11th International Symposium on Radiative Corrections (Applications of Quantum Field Theory to Phenomenology)

22-27 September 2013

Lumley Castle Hotel, Durham, UK

${ }^{*}$ Speaker. 


\section{Introduction}

Top quarks are the heaviest known fundamental particles, and the precise theoretical understanding of their production and decay mechanism, within or beyond the Standard Model, has deep implications on countless aspects of the LHC physics programme. At the LHC, top quarks are mainly produced as $\mathrm{t} \bar{t}$ pairs and via single-top production in the $t$-channel or in the associated $\mathrm{Wt}$ mode. At $8 \mathrm{TeV}$ these latter single-top channels amount to $40 \%$ and $10 \%$ of the $\mathrm{tt}$ cross section, respectively. In spite of their smaller cross sections, they play an important role as direct probes of top-quark weak interactions and of their flavour structure. The separation of top-production into individual top-pair and single-top contributions poses non-trivial experimental and theoretical challenges, which are mainly due to the similarity among the final states associated with the various mechanisms of top-production and decay. In particular, the definition of $t \bar{t}$ and Wt production involves notorious and quite subtle theoretical issues [1].

A theoretically rigorous approach consists of adopting the four-flavour (4F) scheme, where initial-state $b$-quarks result from gluons via explicit $g \rightarrow b \bar{b}$ splittings. In this framework, the process $\mathrm{pp} \rightarrow \mathrm{W}^{+} \mathrm{W}^{-} \mathrm{b} \overline{\mathrm{b}}+X$ provides a unified description of $\mathrm{Wt}$ and $\overline{\mathrm{t}}$ production [2], and the presence of the $\mathrm{t} \overline{\mathrm{t}}-\mathrm{Wt}$ interference at $\mathrm{LO}$ stabilises the perturbative expansion. In the $4 \mathrm{~F}$ scheme, treating finite-top-width effects in the complex-mass scheme [3] ensures a consistent off-shell continuation of top-quark propagators and allows one to include double-, single-, and non-resonant contributions to $\mathrm{pp} \rightarrow \mathrm{W}^{+} \mathrm{W}^{-} \mathrm{b} \overline{\mathrm{b}}+X$ with all relevant interferences. Moreover, the ill-defined separation of top-pair and Wt production can be replaced by a gauge-invariant separation of $\mathrm{pp} \rightarrow \mathrm{W}^{+} \mathrm{W}^{-} \mathrm{b} \overline{\mathrm{b}}$ into its narrow-top-width limit, which corresponds to on-shell top-pair production and decay, and a finite-width remainder that includes off-shell $t \bar{t}$ effects as well as single-top and non-resonant contributions plus related interferences.

The presence of four final-state particles and intermediate top-quark resonances render the simulation of $\mathrm{W}^{+} \mathrm{W}^{-} \mathrm{b} \overline{\mathrm{b}}$ production quite challenging beyond LO. First NLO calculations with massless b-quarks have been presented in [4-6]. However, due to the presence of collinear singularities, phase-space regions with unresolved b-quarks are not accessible in the massless b-quark approximation of [4-6]. To fill this gap, two independent complete $\mathrm{NLO} \mathrm{W}^{+} \mathrm{W}^{-} \mathrm{b} \overline{\mathrm{b}}$ calculations including off-shell W-boson decays and massive b-quarks in the $4 \mathrm{~F}$ scheme have been performed in $[7,8]$. This proceeding contribution is based on the result of [8]. These simulations provide NLO accurate $\mathrm{W}^{+} \mathrm{W}^{-} \mathrm{b} \overline{\mathrm{b}}$ predictions in the full phase space and allow one to investigate, for the first time, top-pair and single-top production in presence of jet vetoes or jet bins, such as in the case of the $\mathrm{H} \rightarrow \mathrm{W}^{+} \mathrm{W}^{-}$analysis.

\section{Technical tools and ingredients of the calculation}

We will focus on NLO predictions for $\mathrm{pp} \rightarrow v_{\mathrm{e}} \mathrm{e}^{+} \mu^{-} \bar{v}_{\mu} \mathrm{b} \overline{\mathrm{b}}$, which comprises $\mathrm{t} \overline{\mathrm{t}}$ production and decay in the opposite-flavour di-lepton channel. For brevity we will denote this reaction as $\mathrm{W}^{+} \mathrm{W}^{-} \mathrm{b} \overline{\mathrm{b}}$ production, keeping in mind that all off-shell and interference effects related to the $v_{\mathrm{e}} \mathrm{e}^{+} \mu^{-} \bar{v}_{\mu}$ final state are consistently handled in the complex-mass scheme [3], where finite-width effects are systematically absorbed in the imaginary part of the renormalised pole mass. The complex-mass scheme is used also for the off-shell continuation of top-quark resonances [5]. Ex- 

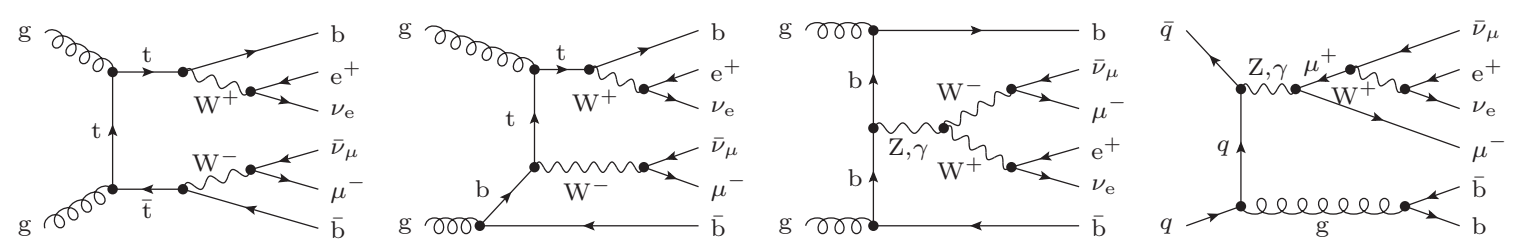

Figure 1: Representative tt-like $\left(1^{\text {st }}\right)$ and $\mathrm{Wt}$-like $\left(2^{\text {nd }}\right)$ tree diagrams, and topologies without top resonances and with two $\left(3^{\text {rd }}\right)$ or only one $\left(4^{\text {th }}\right)$ resonant W-boson.

amples of tree diagrams involving two, one and no top-quark resonances are illustrated in Fig. 1. The second diagram in Fig. 1 is the $4 \mathrm{~F}$-scheme analogon of $t$-channel $\mathrm{gb} \rightarrow \mathrm{tW}^{-}$production in the five-flavour (5F) scheme, and the initial-state $g \rightarrow b \bar{b}$ splitting is related to the b-quark parton distribution in 5F PDFs. At NLO we include the full set of tree, one-loop and real-emission diagrams that contribute to $v_{\mathrm{e}} \mathrm{e}^{+} \mu^{-} \bar{v}_{\mu} \mathrm{b} \overline{\mathrm{b}}$ production without applying any approximation. In particular nonresonant $Z / \gamma \rightarrow v_{\mathrm{e}} \mathrm{e}^{+} \mu^{-} \bar{v}_{\mu}$ sub-topologies like in the fourth diagram of Fig. 1 are included also in the virtual and real corrections. The bottom- and top-quark masses are renormalised in the on-shell scheme, and their contributions are retained everywhere.

The entire calculation has been performed with highly flexible and automated NLO programs, and the high complexity resulting from the presence of multiple top- and W-resonances, as well as from the wide spectrum of involved scales, render $\mathrm{pp} \rightarrow \mathrm{W}^{+} \mathrm{W}^{-} \mathrm{b} \overline{\mathrm{b}}$ an excellent technical benchmark to test the performance of the employed tools. To evaluate tree, virtual, and real-emission amplitudes, we employed OpEnLoops [9], a new one-loop generator that will become public in the next future. The OPENLOOPS program is based on a novel numerical recursion, which is formulated in terms of loop-momentum polynomials called "open loops" and allows for a fast evaluation of scattering amplitudes with many external particles. It uses the COLLIER library [10] for the numerically stable evaluation of tensor integrals [11, 12] and scalar integrals [13]. This proceeding reports on the results of [8], which is, together with $[14,15]$, one of the very first applications of OPENLOOPS. Phase-space integration and infrared subtractions are performed with an in-house NLO Monte-Carlo framework [16], which is interfaced with OPENLOOPS and provides full automation along the entire chain of operations that are required for NLO calculations. This tool is applicable to any Standard-Model process at NLO QCD. Infrared singularities are handled with dipole subtraction [17, 18], and since collinear $\mathrm{g} \rightarrow \mathrm{b} \overline{\mathrm{b}}$ splittings are regularised by the finite b-quark mass, corresponding subtraction terms are not included. The phase-space integrator is based on the adaptive multi-channel technique [19] and implements dedicated channels for the dipole subtraction terms, which improve the convergence, especially for multi-resonance processes. Multiple scale variations in a single run are also supported. This tool has been validated in several NLO processes $[5,20]$, and, in combination with OPENLOOPS and COLLIER, it is also applicable to NNLO calculations [21]. The correctness of the results is supported by various checks: OPENLOOPS has been validated against an independent in-house generator for more than hundred partonic processes, including $\mathrm{W}^{+} \mathrm{W}^{-} \mathrm{b} \bar{b}$ production with massless $b$-quarks and various processes with massive heavy-quarks. For the process at hand we checked the cancellation of infrared and ultraviolet singularities. The correctness of phase-space integration and dipole subtraction was tested by means of a second calculation based on OPENLOOPS in combination with SHERPA [22, 23] and AMEgIC++ [24]. 


\section{Scale choice for top-pair and single-top production}

In order to isolate off-shell and single-top effects associated with the finite top-quark width (FtW) we decompose the differential $\mathrm{W}^{+} \mathrm{W}^{-} \mathrm{b} \overline{\mathrm{b}}$ cross section by means of a numerical extrapolation in the narrow-width limit [5] as

$$
\mathrm{d} \sigma_{\mathrm{W}^{+} \mathrm{W}^{-} \mathrm{b} \overline{\mathrm{b}}}=\mathrm{d} \sigma_{\overline{\mathrm{t}}}+\mathrm{d} \sigma_{\mathrm{W}^{+} \mathrm{W}^{-} \mathrm{b} \overline{\mathrm{b}}}^{\mathrm{FtW}}, \quad \mathrm{d} \sigma_{\overline{\mathrm{tt}}}=\lim _{\Gamma_{\mathrm{t}} \rightarrow 0}\left(\frac{\Gamma_{\mathrm{t}}}{\Gamma_{\mathrm{t}}^{\text {phys }}}\right)^{2} \mathrm{~d} \sigma_{\mathrm{W}^{+} \mathrm{W}^{-} \mathrm{b} \overline{\mathrm{b}}}\left(\Gamma_{\mathrm{t}}\right),
$$

where the factor $\left(\Gamma_{\mathrm{t}} / \Gamma_{\mathrm{t}}^{\text {phys }}\right)^{2}$ compensates the $1 / \Gamma_{\mathrm{t}}^{2}$ scaling of the cross section in such a way that top-decay branching fractions remain constant when $\Gamma_{\mathrm{t}} \rightarrow 0$. By construction the $\mathrm{d} \sigma_{\mathrm{W}^{+} \mathrm{W}^{-} \mathrm{b} \overline{\mathrm{b}}}^{\mathrm{FtW}}$ remainder in (3.1) contains all finite-top-width effects, including off-shell t $\bar{t}$ production as well as single-top and non-resonant contributions.

As compared to $\mathrm{W}^{+} \mathrm{W}^{-} \mathrm{b} \overline{\mathrm{b}}$ production with two hard b-jets, the fully inclusive case involves a much wider spectrum of scales, ranging from $m_{\mathrm{b}}$ to $m_{\mathrm{t}}$. This renders theoretical calculations significantly more involved. In particular, given that the t⿱t and $\mathrm{Wt}$ contributions to $\mathrm{W}^{+} \mathrm{W}^{-} \mathrm{b} \overline{\mathrm{b}}$ production are characterised by very different scales, it is a priori not clear if a conventional QCD scale choice can ensure a perturbatively stable description of both contributions. For t⿱亠t production, a scale of the order of the geometric average of the top-quark transverse energies,

$$
\mu_{\mathrm{tt}}^{2}=E_{\mathrm{T}, \mathrm{t}} E_{\mathrm{T}, \overline{\mathrm{t}}} \quad \text { with } \quad E_{\mathrm{T}, i}^{2}=m_{i}^{2}+p_{\mathrm{T}, i}^{2},
$$

is known to ensure a good perturbative convergence [5]. In the case of the single-top $\mathrm{W}^{-} \mathrm{t}$ contribution one has to deal with two sub-processes: a collinear $g \rightarrow b \bar{b}$ initial-state splitting followed by $\mathrm{gb} \rightarrow \mathrm{W}^{-} \mathrm{t}$ hard scattering. (The charge-conjugate channels are implicitly understood.) The respective characteristic scales are the bottom- and the top-quark transverse energies, $E_{\mathrm{T}, \mathrm{b}} \ll E_{\mathrm{T}, \mathrm{t}}$, and a QCD scale of type

$$
\mu_{\mathrm{tW}}^{2}=E_{\mathrm{T}, \mathrm{t}} E_{\mathrm{T}, \overline{\mathrm{b}}}
$$

should represent an appropriate choice, since $\alpha_{\mathrm{s}}^{2}\left(\mu_{\mathrm{tW}}^{2}\right) \simeq \alpha_{\mathrm{s}}\left(E_{\mathrm{T}, \mathrm{t}}^{2}\right) \alpha_{\mathrm{s}}\left(E_{\mathrm{T}, \overline{\mathrm{b}}}^{2}\right)$ guarantees that the $\alpha_{\mathrm{s}}$ factor associated with the collinear $\mathrm{g} \rightarrow \mathrm{b} \overline{\mathrm{b}}$ splitting is effectively evaluated at the scale $E_{\mathrm{T}, \mathrm{b}}$, similarly as in the resummation of initial-state b-quark emissions in the evolution of 5F PDFs. Vice versa, using a global QCD scale of the order $m_{\mathrm{t}}$ might underestimate the single-top component of $\mathrm{pp} \rightarrow \mathrm{W}^{+} \mathrm{W}^{-} \mathrm{b} \bar{b}$ by up to a factor $\alpha_{\mathrm{s}}\left(m_{\mathrm{b}}\right) / \alpha_{\mathrm{s}}\left(m_{\mathrm{t}}\right) \sim 2$ at LO. This would be compensated by $\ln \left(m_{\mathrm{b}}\right)-$ enhanced higher-order corrections, resulting in a poor perturbative convergence. For an accurate description of the single-top contribution, the above considerations motivate a dynamic QCD scale that interpolates between (3.2) and (3.3) in $\mathrm{t} \overline{\mathrm{t}}$ - and Wt-dominated regions, respectively. Such a scale can be defined as

$$
\mu_{\mathrm{WWbb}}^{2}=\mu_{\mathrm{W}+\mathrm{b}} \mu_{\mathrm{W}-\overline{\mathrm{b}}} \quad \text { with } \quad \mu_{\mathrm{Wb}}=P_{\mathrm{b}}\left(p_{\mathrm{W}}, p_{\mathrm{b}}\right) E_{\mathrm{T}, \mathrm{b}}+P_{\mathrm{t}}\left(p_{\mathrm{W}}, p_{\mathrm{b}}\right) E_{\mathrm{T}, \mathrm{t}}
$$

where $\mathrm{Wb}$ represents either $\mathrm{W}^{+} \mathrm{b}$ or $\mathrm{W}^{-} \overline{\mathrm{b}}$, and the functions $P_{\mathrm{b}}$ and $P_{\mathrm{t}}=1-P_{\mathrm{b}}$ describe the probability that the b-quark of a given $\mathrm{Wb}$ pair arises from an initial-state $\mathrm{g} \rightarrow \mathrm{b} \overline{\mathrm{b}}$ splitting or from a $\mathrm{t} \rightarrow \mathrm{Wb}$ decay, respectively. Their approximate functional form can be obtained from the leading 


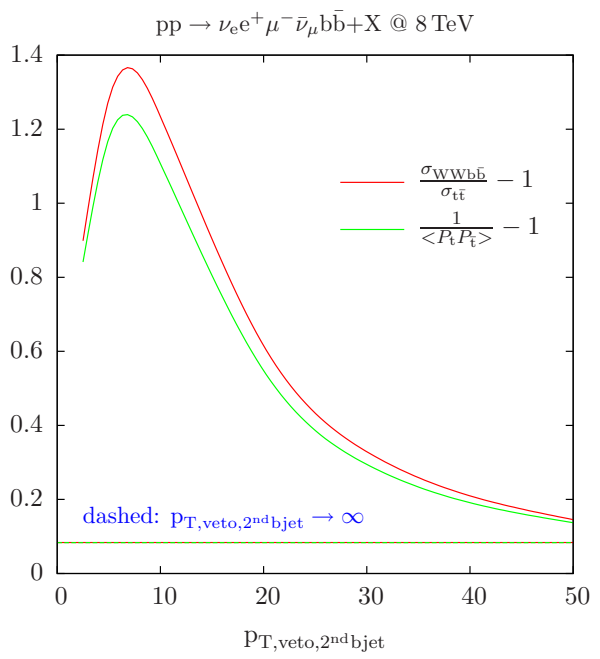

Figure 2: The relative size of finite-top-width corrections at LO is compared to the ratio of the corresponding expectation values of the non- $\bar{t} \bar{t}$ and $t \bar{t}$ probabilities at $\mathrm{LO}$, in dependence of a $p_{\mathrm{T}, \text { veto }}$ on the second $b$-jet. Their full agreement in the limit $\mathrm{p}_{\mathrm{T}, \text { veto }, 2^{\text {nd }} \text { bjet }} \rightarrow \infty$ essentially reflects the contruction (3.6) of the scale choice $\mu_{\mathrm{WWbb}}$.

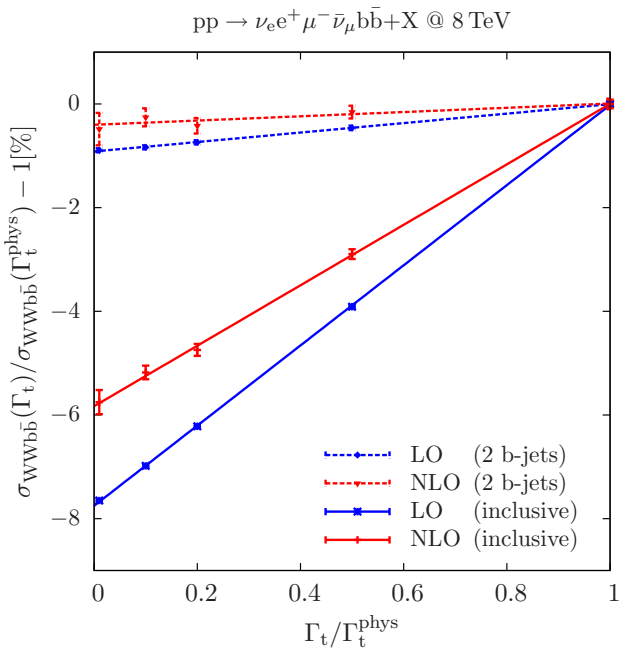

Figure 3: Numerical extrapolation of the LO and $\mathrm{NLO} \mathrm{W}^{+} \mathrm{W}^{-} \mathrm{b} \overline{\mathrm{b}}$ cross section with leptonic cuts in the narrow-top-width limit, $\Gamma_{\mathrm{t}} \rightarrow 0$. Results are shown as relative deviations (in percent) with respect to the $\mathrm{W}^{+} \mathrm{W}^{-} \mathrm{b} \overline{\mathrm{b}}$ cross section with $\Gamma_{\mathrm{t}}=$ $\Gamma_{\mathrm{t}}^{\text {phys }}$. Results with inclusive jet emission are compared to a $\bar{t} \bar{t}$-signal analysis with two b-jets.

matrix-element singularities associated with the $\mathrm{g} \rightarrow \mathrm{b} \overline{\mathrm{b}}$ and $\mathrm{t} \rightarrow \mathrm{Wb}$ sub-processes and the unitarity requirement $P_{\mathrm{t}}+P_{\mathrm{b}}=1$,

$$
\chi_{\mathrm{b}}=\frac{m_{\mathrm{t}}^{2}}{E_{\mathrm{T}, \mathrm{b}}^{2}}, \quad \chi_{\mathrm{t}}=\frac{m_{\mathrm{t}}^{4}}{\left[\left(p_{\mathrm{W}}+p_{\mathrm{b}}\right)^{2}-m_{\mathrm{t}}^{2}\right]^{2}+\Gamma_{\mathrm{t}}^{2} m_{\mathrm{t}}^{2}}, \quad P_{\mathrm{b}}=1-P_{\mathrm{t}}=\frac{\chi_{\mathrm{b}}}{\chi_{\mathrm{b}}+R \chi_{\mathrm{t}}} .
$$

The constant $R$ can be derived from the condition

$$
\int \mathrm{d} \sigma_{\mathrm{W}^{+} \mathrm{W}^{-} \mathrm{b} \overline{\mathrm{b}}}^{\mathrm{Ft}}=\int \mathrm{d} \Phi\left[1-P_{\mathrm{t}}(\Phi) P_{\mathrm{t}}(\Phi)\right] \frac{\mathrm{d} \sigma_{\mathrm{W}^{+} \mathrm{W}^{-} \mathrm{b} \overline{\mathrm{b}}}}{\mathrm{d} \Phi},
$$

i.e. by requiring that finite-top-width corrections to the inclusive $\mathrm{W}^{+} \mathrm{W}^{-} \mathrm{b} \overline{\mathrm{b}}$ cross section correspond to the contribution from non-t $\overline{\mathrm{t}}$ events according to the probability distributions $P_{\mathrm{b}}$ and $P_{\mathrm{t}}$. The tuning of $R$ is performed in LO approximation on the fully inclusive level and yields $R=7.96$. As a confirmation of the resulting scale choice, in Fig. 2 we perform a LO comparison of the relative size of finite-top-width corrections and the corresponding probability ratio, in dependence of a $p_{\mathrm{T}, \text { veto }}$ on the second $\mathrm{b}$-jet. This veto is chosen as it enhances the relative Wt contribution and thereby provides access to the phase-space region we are aiming for. For details on the implementation of this scale choice at NLO we refer to [8].

\section{Predictions for the $\mathrm{LHC}$ at $8 \mathrm{TeV}$}

In the following we present predictions for $\mathrm{pp} \rightarrow \mathrm{W}^{+} \mathrm{W}^{-} \mathrm{b} \overline{\mathrm{b}}$ at $8 \mathrm{TeV}$ in presence of the leptonic cuts $p_{\mathrm{T}, \ell}>20 \mathrm{GeV},\left|\eta_{\ell}\right|<2.5, p_{\mathrm{T}, \text { miss }}>20 \mathrm{GeV}$. Detailed setup and input parameters can be found in [8]. The renormalisation and factorisation scales are set to $\mu_{\mathrm{R}, \mathrm{F}}=\xi_{\mathrm{R}, \mathrm{F}} \mu_{0}$, with $\mu_{0}=\mu_{\mathrm{Wwbb}}$, 

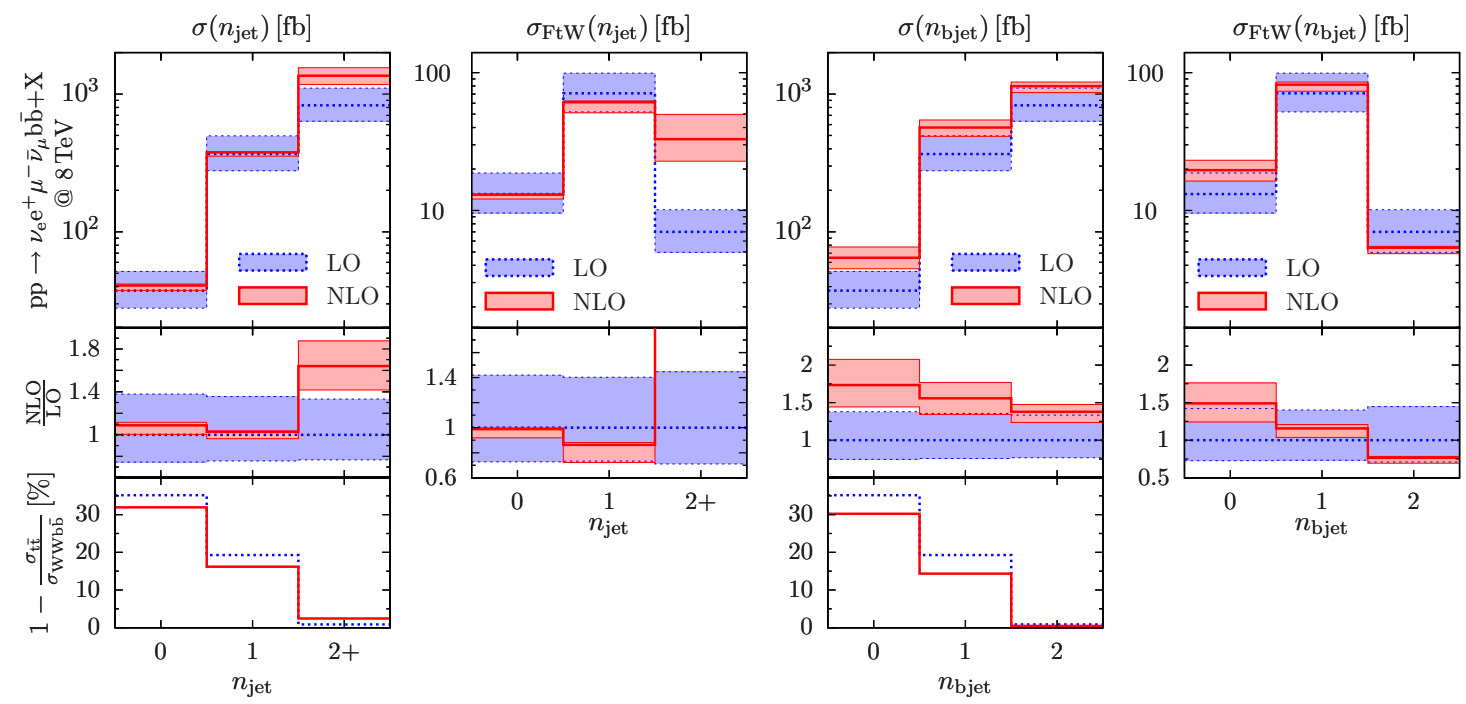

Figure 4: $\mathrm{LO}$ and NLO predictions for $\mathrm{pp} \rightarrow \mathrm{W}^{+} \mathrm{W}^{-} \mathrm{b} \overline{\mathrm{b}}$ at $8 \mathrm{TeV}$ with scale variations and corrections, NLO/LO, for $\mu_{0}=\mu_{\mathrm{Wwbb}}$ are shown for the bins with 0,1 , and $\geq 2$ jets (left plots) and 0,1 , and $2 \mathrm{~b}$-jets (right plots). The $2^{\text {nd }}\left(4^{\text {th }}\right)$ plot depicts the finite-top-width contributions, whose relative size is indicated in the lowest panel of the $1^{\text {st }}\left(3^{\text {rd }}\right)$ plot.

where $\xi_{\mathrm{R}}=\xi_{\mathrm{F}}=1$ corresponds to the default scale choice. Theoretical uncertainties are assessed by applying the scale variations $\left(\xi_{\mathrm{R}}, \xi_{\mathrm{F}}\right)=(2,2),(2,1),(1,2),(1,0.5),(0.5,1),(0.5,0.5)$.

Fig. 3 illustrates the extrapolation of the $\mathrm{W}^{+} \mathrm{W}^{-} \mathrm{b} \overline{\mathrm{b}}$ cross section in the narrow-top-width limit (3.1). The results are well consistent—at the few-permil level—with the expected linear convergence of the NLO cross section in the $\Gamma_{\mathrm{t}} \rightarrow 0$ limit. This provides a non-trivial check of the consistency of the calculation, since the narrow-width limit involves delicate cancellations of logarithmic singularities that arise from virtual and real soft-gluon corrections to the resonant top-quark propagators. Finite-width effects turn out to be at the sub-percent level if one requires the presence of two b-jets, like in a typical tit-signal analysis. For the total cross section they are instead clearly more important. Their net effect, which results from the interplay of negative off-shell corrections and positive single-top contributions, amounts to about $+6 \%(8 \%)$ at NLO(LO).

Predictions for the cross sections in exclusive (b-)jet bins are depicted in Fig. 4 for the same scale choice $\mu_{0}=\mu_{\mathrm{WWbb}}$. (As in [8], (b-)jets are defined by the requirements $p_{\mathrm{T},(\mathrm{b}-) \text { jet }}>30 \mathrm{GeV}$ and $\left|\eta_{(\mathrm{b}-) \text { jet }}\right|<2.5$.) Detailed numbers and a comparison to a fixed scale $\mu_{0}=m_{\mathrm{t}}$ are provided in [8]. For the total cross section, we find positive corrections of around $40 \%$. Scale uncertainties decrease from about $30 \%$ at $\mathrm{LO}$ to $10 \%$ at NLO. The different jet bins receive quite different corrections, indicating that a significant fraction of the 0 - and 1 -jet bin cross sections migrates to the inclusive 2-jet bin. We attribute this feature to the rather high probability of light-jet emissions with $p_{\mathrm{T}} \gtrsim 30 \mathrm{GeV}$. While NLO scale uncertainties turn out to be fairly small in all jet bins, matching to the parton shower is certainly important for a more reliable description of such radiative processes. Finite-top-width (FtW) contributions are shown in the second plot of Fig. 4. For what concerns the total $\mathrm{W}^{+} \mathrm{W}^{-} \mathrm{b} \overline{\mathrm{b}}$ cross section their impact is around $6 \%$, and the scale $\mu_{\mathrm{Wwbb}}$ guarantees a good perturbative convergence. The results suggest that $\mathrm{FtW}$ effects are dominated by a single-top 

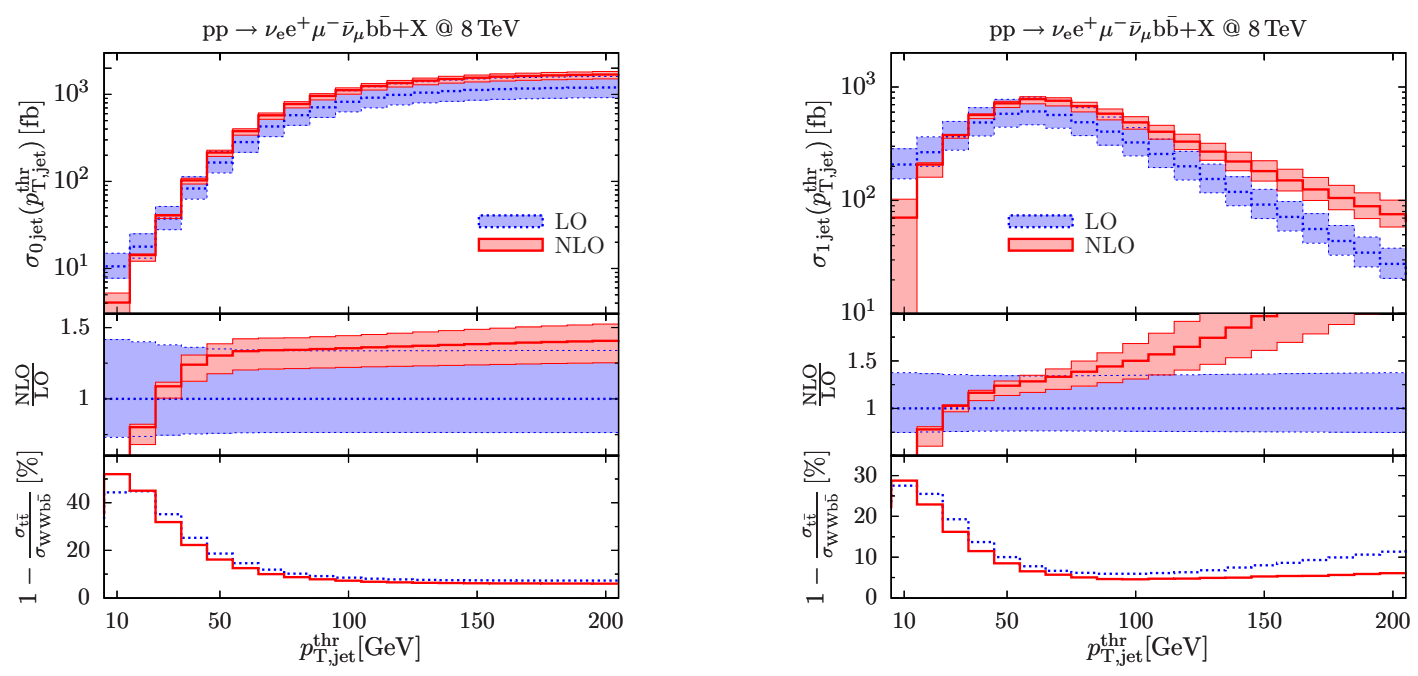

Figure 5: $\mathrm{LO}$ and $\mathrm{NLO} \mathrm{W}^{+} \mathrm{W}^{-} \mathrm{b} \overline{\mathrm{b}}$ cross sections in the exclusive bins with $N_{j}=0$ (left) and $N_{j}=1$ (right) jets as functions of the jet- $p_{\mathrm{T}}$ threshold, $p_{\mathrm{T}, \mathrm{jet}}^{\text {thr }}$. The middle of each bin corresponds to the actual value of $p_{\mathrm{T}, \mathrm{jet}}^{\mathrm{thr}}$. The central and lower frames show the $K$-factor and the relative impact in percent of finite-top-width contributions. Where depicted, bands correspond to independent scale variations of $\mu_{\mathrm{R}, \mathrm{F}}$ by a factor of two around the central scale $\mu_{\mathrm{Wwbb}}$, not taking into account antipodal variations.

Wt component, which is concentrated in the 1-jet bin at $\mathrm{LO}$ and tends to migrate to the 2-jet bin due to light-jet emissions at NLO. The fact that the FtW part of the 2-jet bin features a 40-50\% NLO uncertainty is irrelevant, since this contribution represents less than $3 \%$ of the complete cross section in the 2-jet bin. In the 0- and 1-jet bins, whose FtW components amount to 32\% and 16\%, respectively, NLO scale uncertainties are as small as $10 \%$.

In the right plots of Fig. 4 we report analogous results for the $\mathrm{W}^{+} \mathrm{W}^{-} \mathrm{b} \overline{\mathrm{b}}$ cross section and its $\mathrm{FtW}$ contribution in b-jet bins. As compared to the case of generic jets, we observe that $\mathrm{W}^{+} \mathrm{W}^{-} \mathrm{b} \overline{\mathrm{b}}$ $\mathrm{K}$-factors feature a less pronounced dependence on the b-jet multiplicity. This is due to the fact that NLO emissions consist of light jets and are thus less likely to induce bin migrations in the case of b-jet bins. Scale uncertainties at NLO are at the 20\%, 15\% and $10 \%$ level in the bins with 0,1 , and 2 b-jets, respectively. Finite-top-width contributions turn out to be even more stable than full $\mathrm{W}^{+} \mathrm{W}^{-} \mathrm{b} \overline{\mathrm{b}}$ results with the scale $\mu_{\mathrm{Wwbb}}$. Although the scale $m_{\mathrm{t}}$ tends to give slightly larger uncertainties, in general, jet- and b-jet-bin results indicate that the conventional scale $\mu_{0}=m_{\mathrm{t}}$ yields a similarly good perturbative convergence as $\mu_{0}=\mu_{\mathrm{Wwbb}}$ [8]. However, it is a priori not clear if this holds also for more exclusive observables.

To illustrate jet-veto and jet-binning effects in more detail, in Fig. 5 we plot the integrated $\mathrm{W}^{+} \mathrm{W}^{-} \mathrm{b} \overline{\mathrm{b}}$ cross section in exclusive bins with $N_{j}=0$ and $N_{j}=1$ jets versus the $p_{\mathrm{T}}$-threshold that defines jets. (The $\left|\eta_{(\mathrm{b}-) \text { jet }}\right|<2.5$ requirement for (b-)jets is understood.) The 0 -jet bin corresponds to the integrated cross section in presence of a jet veto, $p_{\mathrm{T} \text {,jet }}<p_{\mathrm{T}, \mathrm{jet}}^{\mathrm{thr}}$. At large $p_{\mathrm{T}, \mathrm{jet}}^{\text {thr }}$ the $K$-factor and the FtW contributions converge quite smoothly towards their inclusive limit. In contrast, the region of small transverse momentum features a very pronounced dependence on $p_{\mathrm{T}, \mathrm{jet}}^{\text {thr }}$ : FtW corrections grow from $6 \%$ up to more than $40 \%$, and the $K$-factor decreases very fast due to the presence of 

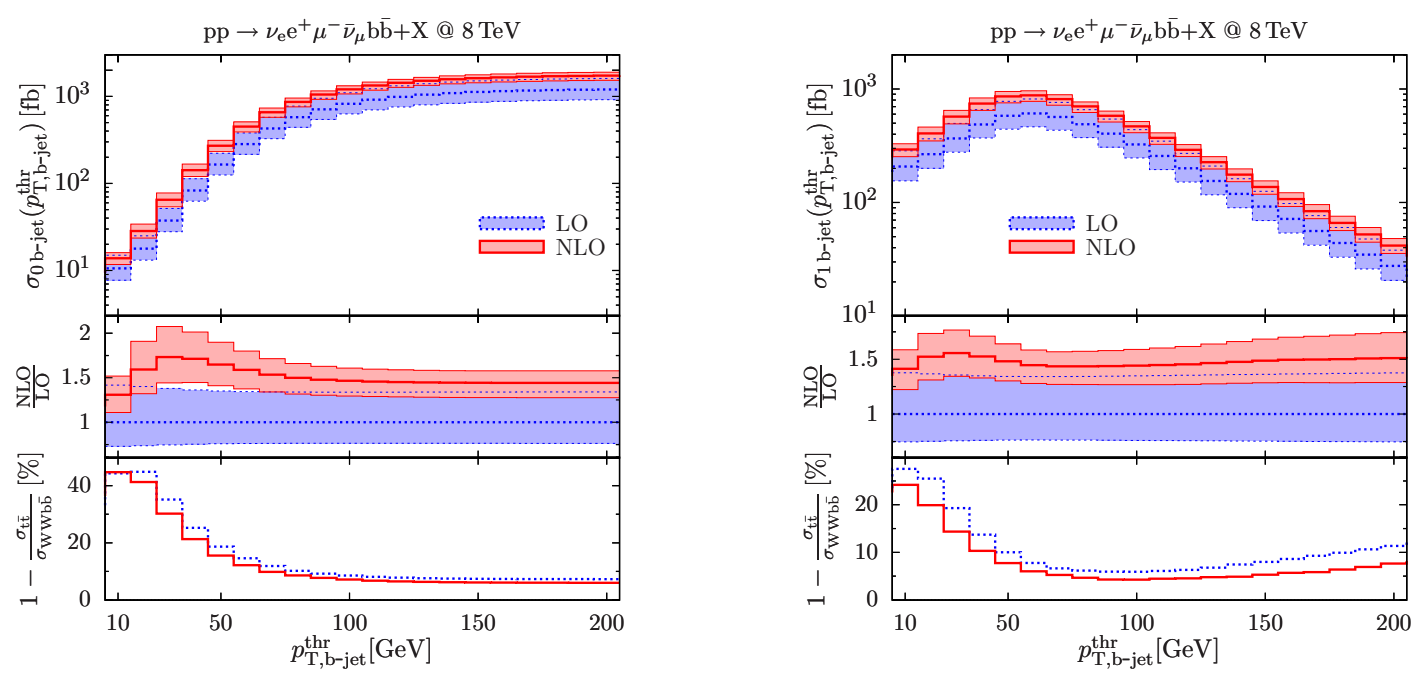

Figure 6: $\mathrm{LO}$ and $\mathrm{NLO} \mathrm{W}^{+} \mathrm{W}^{-} \mathrm{b} \overline{\mathrm{b}}$ cross sections in the exclusive bins with $N_{\mathrm{b}}=0$ (left) and $N_{\mathrm{b}}=1$ (right) b-jets versus the b-jet- $p_{\mathrm{T}}$ threshold. Same conventions as in Fig. 5 .

a soft singularity at $p_{\mathrm{T}, \mathrm{jet}}^{\mathrm{thr}} \rightarrow 0$. For a jet veto with $p_{\mathrm{T}, \mathrm{jet}}^{\mathrm{thr}}=30 \mathrm{GeV}$ we observe a $98 \%$ suppression of the $\mathrm{W}^{+} \mathrm{W}^{-} \mathrm{b} \bar{b}$ cross section. Yet the moderate size of the $K$-factor and NLO scale variations indicates that the perturbative expansion is still rather stable in this regime. In the 1-jet bin, the limit of small $p_{\mathrm{T}, \text { jet }}^{\mathrm{thr}}$ is driven by the effect of the veto on the second jet, and NLO and FtW corrections behave rather similarly as for the 0 -jet bin in this region. In the opposite regime, $p_{\mathrm{T}, \mathrm{jet}}^{\mathrm{thr}}$ mainly acts as a lower $p_{\mathrm{T}}$ bound for the first jet, and $\bar{t}$ production with LO on-shell kinematics turns out to be kinematically disfavoured at large $p_{\mathrm{T}, \mathrm{jet}}^{\mathrm{thr}}$, while the relative importance of NLO jet emission and FtW effects increases quite dramatically.

Analogous results for exclusive bins with $N_{\mathrm{b}}=0$ and $N_{\mathrm{b}}=1$ b-jets are displayed in Fig. 6 . In this case the reduced sensitivity of b-jet bins to NLO real emission is clearly reflected in the much better stability of the $K$-factor with respect to variations of $p_{\mathrm{T}, \mathrm{bjet}}^{\mathrm{thr}}$. Similarly as for jet bins, $\mathrm{FtW}$ corrections are strongly enhanced at small $p_{\mathrm{T}}$. This effect can be attributed to the single-top $\mathrm{Wt}$ channels, and the inclusion of $\mathrm{t} \bar{t}-\mathrm{Wt}$ interferences, as in the present $\mathrm{W}^{+} \mathrm{W}^{-} \mathrm{b} \overline{\mathrm{b}}$ calculation, is clearly advisable in this regime.

\section{Summary and conclusions}

We have reported on a complete NLO simulation of $\mathrm{W}^{+} \mathrm{W}^{-} \mathrm{b} \overline{\mathrm{b}}$ production at the $\mathrm{LHC}$, including W-boson decays in the opposite-flavour di-lepton channel, finite $\mathrm{W}$ - and top-width effects, and massive b-quarks in 4F scheme. The finite b-quark mass acts as a regulator of collinear singularities and allows one to describe the full b-quark phase space, including single-top contributions that arise from initial-state $\mathrm{g} \rightarrow \mathrm{b} \overline{\mathrm{b}}$ splittings followed by $\mathrm{gb} \rightarrow \mathrm{Wt}$ scattering. This yields a gauge-invariant description of top-pair, single-top, and non-resonant $\mathrm{W}^{+} \mathrm{W}^{-} \mathrm{b} \overline{\mathrm{b}}$ production including all interferences at NLO QCD. We introduced a dynamical scale choice aimed at an improved perturbative stability of initial-state $\mathrm{g} \rightarrow \mathrm{b} \overline{\mathrm{b}}$ splittings in single-top contributions. Using this scale, the NLO 
$\mathrm{W}^{+} \mathrm{W}^{-} \mathrm{b} \overline{\mathrm{b}}$ cross section in bins with 0,1 and 2 jets features NLO scale uncertainties at the $10-15 \%$ level. The more conventional choice $\mu_{0}=m_{\mathrm{t}}$ yields similarly small NLO uncertainties in jet bins. Finite-top-width corrections mainly originate from single-top and off-shell $t \bar{t}$ contributions. They represent $6 \%$ of the integrated cross section and are strongly sensitive to the jet multiplicity. In the 2-jet bin they are as small as $2 \%$, while in the 1- and 0 -jet bins they reach the $16 \%$ and $32 \%$ level, respectively. Also NLO corrections vary quite strongly with the jet multiplicity. Moreover, finite-top-width contributions receive quite different corrections as compared to on-shell tt production. The non-trivial interplay of NLO and finite-width effects is especially relevant for the 0 - and 1 -jet bins. It plays an important role for the accurate description of associated Wt production, as well as for top-backgrounds to $\mathrm{H} \rightarrow \mathrm{W}^{+} \mathrm{W}^{-}$and to other searches based on leptons, large missing energy and jet vetoes. All employed tools are fully automated and can be easily exploited to extend the present results to the like-flavour di-lepton channel or to simulate any other Standard-Model process at NLO QCD.

\section{Acknowledgments}

Our research is funded by the SNSF and supported, in part, by the European Commission through the network PITN-GA-2010-264564 (LHCPhenoNet).

\section{References}

[1] C. D. White, S. Frixione, E. Laenen, and F. Maltoni, JHEP 0911, 074 (2009), 0908 . 0631.

[2] N. Kauer and D. Zeppenfeld, Phys.Rev. D65, 014021 (2002), hep-ph/0107181.

[3] A. Denner et al., Nucl.Phys. B724, 247 (2005), hep-ph/ 0505042.

[4] A. Denner et al., Phys.Rev.Lett. 106, 052001 (2011), 1012.3975.

[5] A. Denner et al., JHEP 1210, 110 (2012), 1207.5018.

[6] G. Bevilacqua et al., JHEP 1102, 083 (2011), 1012 . 4230.

[7] R. Frederix (2013), 1311. 4893.

[8] F. Cascioli, S. Kallweit, P. Maierhoefer, and S. Pozzorini (2013), 1312.0546.

[9] F. Cascioli et al., Phys.Rev.Lett. 108, 111601 (2012), 1111.5206.

[10] A. Denner, D. Dittmaier, and L. Hofer, in preparation.

[11] A. Denner and S. Dittmaier, Nucl. Phys. B658, 175 (2003).

[12] A. Denner and S. Dittmaier, Nucl. Phys. B734, 62 (2006).

[13] A. Denner and S. Dittmaier, Nucl. Phys. B844, 199 (2011).

[14] F. Cascioli et al. (2013), 1309.0500. 
[15] F. Cascioli et al. (2013), 1309.5912.

[16] A fully automated C++ Monte-Carlo generator for NLO QCD by S. Kallweit.

[17] S. Catani and M. Seymour, Nucl.Phys. B485, 291 (1997), hep-ph / 9605323.

[18] S. Catani et al., Nucl.Phys. B627, 189 (2002), hep-ph / 0201036.

[19] R. Kleiss and R. Pittau, Comput. Phys. Commun. 83, 141 (1994).

[20] A. Denner, L. Hosekova, and S. Kallweit, Phys.Rev. D86, 114014 (2012), 1209.2389.

[21] M. Grazzini, S. Kallweit, D. Rathlev, and A. Torre (2013), 1309.7000.

[22] T. Gleisberg and F. Krauss, Eur.Phys.J. C53, 501 (2008), 0709.2881.

[23] T. Gleisberg et al., JHEP 0902, 007 (2009), 0811 . 4622.

[24] F. Krauss, R. Kuhn, and G. Soff, JHEP 0202, 044 (2002), hep-ph / 0109036. 